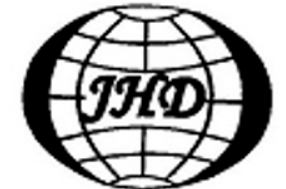

www.sciencedirect.com science/journal/10016058

\title{
The hydrodynamic interactions of an array of truncated circular cylinders as each cylinder oscillates independently with different prescribed modes"
}

\author{
ZENG Xiao-hui, TANG Si-yi \\ Institute of Mechanics, Chinese Academy of Sciences, Beijing 100190, China, E-mail: zxh@imech.ac.cn
}

(Received March 4, 2012, Revised August 5, 2012)

\begin{abstract}
An analytical method based on the eigenfunction expansion and the Graf's addition theorem for Bessel functions is developed to study the hydrodynamic interactions of an array of truncated circular cylinders with each cylinder oscillating independently in different prescribed modes. The hydrodynamic radiation and diffraction of linear waves by such an array of cylinders are investigated and the analytical solutions of the velocity potentials are obtained. After comparisons for degenerated cases and program verifications, several cases for an array of truncated cylinders with each cylinder oscillating independently in surge, sway, heave, roll, and pitch modes with different prescribed amplitudes, are studied and the hydrodynamic forces and moments acting on the cylinders are obtained.
\end{abstract}

Key words: array of truncated circular cylinders, independent oscillation, radiation, hydrodynamic interaction

\section{Introduction}

Many kinds of offshore structures were developed for various exploration and exploitation purposes. The arrays of truncated circular cylinders are the main components of many offshore floating structures $^{[1-6]}$. There are different configurations of cylinders, thus the arrays of cylinders can be classified into two categories: one is the array with all cylinders oscillating as a whole (hereafter referred to as "C1 array"), the other is that with each cylinder in the array oscillating independently (hereafter referred to as " $\mathrm{C} 2$ array"). The interaction between the water waves and the arrays of cylinders is important and was much studied. Most investigations concerned with the " $\mathrm{C} 1$ array". For the "C2 array", the analyses (especially by using the analytical method) were relatively few.

For offshore structures with an irregular configuration, various numerical methods might be the only way to obtain the detailed solution. On the other hand,

* Project supported by the National Natural Science foundation of China (Grant Nos. 11072246, 10702073), the National High Technology Research and Development Program of China (863 Program, Grant No. 2006AA09Z350). Biography: ZENG Xiao-hui (1972- ), Male, Ph. D., Associate Professor for other structures with a regular configuration, such as a single truncated circular cylinder or arrays of circular cylinders, an analytical analysis might be possible. Many studies in this direction based on the eigenfunction expansion were carried out. Sabuncu and Calisal ${ }^{[7]}$ investigated the radiation problem of a single truncated cylinder and calculated the hydrodynamic coefficients. Bhatta and Rahman ${ }^{[8]}$ presented analytical solutions for the velocity potential and wave loads for the case of a vertical circular cylinder with surge, heave and pitch motions in a finite-depth water in the presence of an incident wave. Bhatta ${ }^{[9]}$ presented the computational results of analytical expressions in Ref.[8]. Zheng et al. ${ }^{[10]}$ investigated the radiation and diffraction problem for the interaction of linear water waves with two vertical truncated cylinders. For arrays of cylinders, hydrodynamic interactions between waves and cylinders should be considered. Large-spacing approximation was adopted in some studies, which ignores the evanescent waves attenuating rapidly. Besides, some accurate interaction theories were developed. Kagemoto and $\mathrm{Yue}^{[11]}$ proposed an interaction theory for multiple bodies based on the matrix method and a multiple-scattering idea, by using only the diffraction characteristics of individual members. Yilmaz et al. ${ }^{[12]}$ obtained analytical solutions for group of circular cylinders by using the interaction theory developed in Ref.[11]. Miao et al. ${ }^{[13]}$ 
established a recursion formula for the wave diffraction problems for a huge number of circular piles. Duclos and Clement ${ }^{[14]}$ considered arrays of unevenly spaced cylinders, for the effect of a small level of disorder on the wave transmission. Walker and Eatock Taylor ${ }^{[15]}$ extended the linear diffraction theory for an array of cylinders by considering the incident wave as NewWaves. Walker et al. ${ }^{[16]}$ studied the diffraction of monochromatic waves by an array of four cylinders using the linear and second order theory. Siddorn and Eatock Taylor ${ }^{[17]}$ investigated the independent radiation by an array of cylinders using an exact algebraic method, and numerical results are obtained for hydrodynamic forces acting on each cylinder oscillating in translational modes.

The studies of the hydrodynamic interaction between cylinders were mainly focused on " $\mathrm{C} 1$ array", namely, with all cylinders oscillating as a whole. On the other hand, for " $\mathrm{C} 2$ array", in which each cylinder may oscillate independently, the studies (especially by using analytical methods) were relatively few. The published results for " $\mathrm{C} 2$ array" mainly concern with concrete examples in which each cylinder oscillates in translational modes such as surge, sway and heave, rather than an array oscillate independently in rotational modes such as roll and pitch.

In this paper, the linear water wave radiation and diffraction by an array of truncated circular cylinders, with each cylinder oscillating in arbitrary rigid modes (including translational and rotational modes with any prescribed amplitude), are investigated. An analytical method is proposed for this problem by extending the interaction theory of Kagemoto and Yue ${ }^{[1]}$. Formulas for velocity potentials, hydrodynamic forces and moments are derived, which are applicable to any number, spacing and dimension of cylinders. And numerical results are presented for an array of cylinders in which each cylinder oscillates with prescribed amplitudes in all rigid modes such as surge, sway, heave, roll and pitch. There is no need for considering the yaw because of the axial symmetry of the circular cylinder. This paper includes four sections. In Section 1, the formulas of first order velocity potentials for the hydrodynamic interactions of an array of cylinders, with each cylinder oscillating in different modes (including the rotational mode), are derived. In Section 2, the formulas for the hydrodynamic pressure, the force and the moment are obtained. In Section 3, degenerated cases are calculated for verification. The comparisons show a good agreement. Furthermore, an array of four cylinders is considered, three cases with different oscillation modes are computed. Numerical results are presented and discussed. The last section is the conclusion, focusing on some key points of this paper.

\section{The solutions for velocity potential}

The radiation and diffraction of linear waves by an array of truncated circular cylinders are studied, in which each cylinder oscillates independently in different modes. This means that any cylinder among the array may oscillate with different prescribed amplitude in different mode (such as surge, sway, heave, roll, pitch, and yaw). The sketch of an array of cylinders, the motion modes, and the coordinates systems are shown in Fig.1. Due to the axial symmetry of the circular cylinder, the yaw motion does not introduce hydrodynamic forces for a perfect fluid.
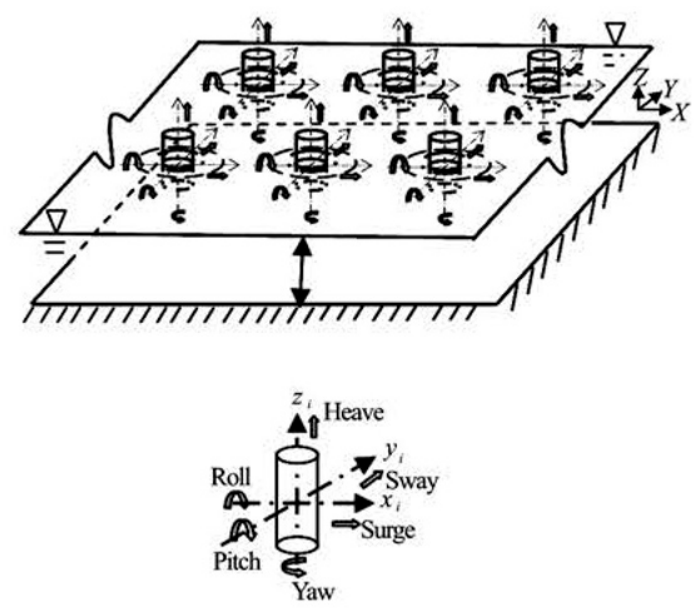

Fig.1 The sketch of an array of cylinders
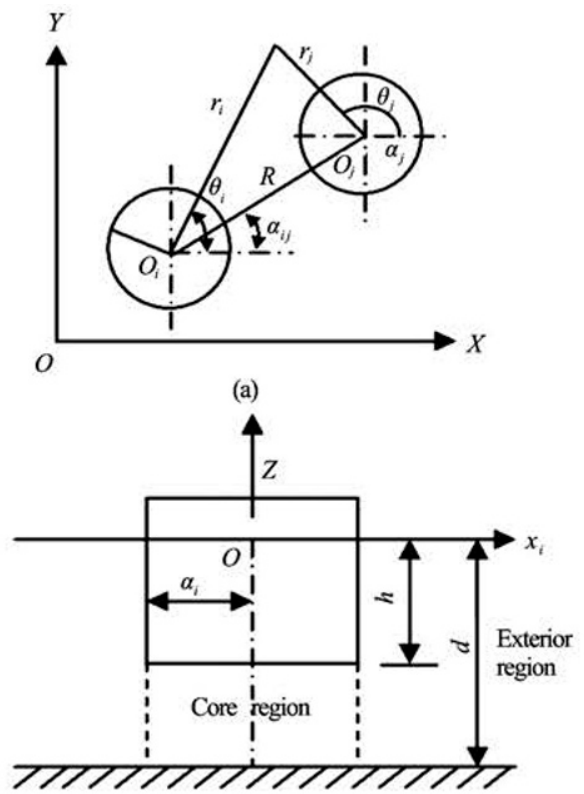

(b)

Fig.2 A truncated circular cylinder array and the local cylindrical coordinate systems 
The array of cylinders consists of $N$ vertical surface-piercing circular cylinders, as shown in Fig.1. A right-handed Cartesian space fixed coordinate system $O X Y Z$ is introduced. The plane $O X Y$ coincides with the undisturbed calm water surface, and the positive $Z$ - axis is pointing vertically upwards.

The local cylindrical coordinate systems $O_{i} r_{i} \theta_{i} z$ are centered on the origin of each cylinder, as shown in Fig.2(a). The coordinates of the origin of cylinder $j$ in the cylindrical coordinate systems centered on the origin of cylinder $i$ are $\left(R_{i j}, \alpha_{i j}, z\right)$. As illustrated in Fig.2(b), the water depth is $d$, the radius and draught of each cylinder are $a_{i}$ and $h_{i}$, respectively. The fluid is divided into two regions: the exterior region and the core region.

In this paper, the following assumptions are adopted: the fluid is incompressible, inviscid and the flow is irrotational. Then the potential theory can be employed. The wave steepness and the amplitude of the motion are small. The linear steady-state oscillations are considered, in which the related physical quantities are periodic functions of the time with angular frequency $\omega_{0}$. Then the space and time factors of the total velocity potential $\Phi_{R D}$ can be separated as

$$
\Phi_{R D}(X, Y, Z, t)=\operatorname{Re}\left[\varphi_{R D}(X, Y, Z) \mathrm{e}^{-\mathrm{i} \omega_{0} t}\right]
$$

The velocity potential $\varphi_{R D}$ may be divided into two parts: one part is the radiation potential generated by the prescribed oscillation of each cylinder, the other one is the velocity potential of the diffraction of emission waves (of other cylinders) by each cylinder. Therefore the potential $\varphi_{R D}$ for an array of cylinders with different prescribed motions may be expressed as the sum of radiation and diffraction potentials mention above

$$
\varphi_{R D}=\varphi_{R}+\varphi_{D 1}=\sum_{i=1}^{N}\left(\sum_{s=1}^{5} \varphi_{R s}^{i}+\varphi_{D 1}^{i}\right)
$$

For a circular truncated cylinder, all the five rigid motions such as surge, sway, heave, roll, and pitch are considered in this paper. The five modes are denoted by $s=1,2,3,4,5 . \varphi_{R s}^{i}$ is the radiation potential of cylinder $i$ in the sth mode oscillation. $\varphi_{D 1 s}^{i}$ is the diffraction potential by cylinder $i$ in the ambient waves emanating from other cylinders. $\varphi_{R}$ is the total radiation potential, and $\varphi_{D 1}$ the total diffraction potential.

The instantaneous displacement of cylinder $i$ in a small-amplitude periodic oscillation with angular frequency $\omega_{0}$ is
$\Xi_{s}^{i}(t)=\operatorname{Re}\left\{\zeta_{s}^{i} \mathrm{e}^{-\mathrm{i} \omega_{0} t}\right\}$

where $\zeta_{s}^{i}$ is the oscillation amplitude of mode $s$ for cylinder $i$. The corresponding velocity is

$\dot{\Xi}_{s}^{i}(t)=\operatorname{Re}\left\{-\mathrm{i} \omega_{0} \zeta_{s}^{i} \mathrm{e}^{-\mathrm{i} \omega_{0} t}\right\}$

The velocity potential should satisfy the Laplace's equation together with the free surface condition, the impermeable condition on the body surface and seabed, and the radiation condition at infinity. The velocity potential $\varphi_{R s-E}^{i}$ for the exterior region and the potential $\varphi_{R s-C}^{i}$ for the core region satisfy the impenetrable body surface conditions as follows

$$
\begin{gathered}
\frac{\partial \varphi_{R s-E}^{i}}{\partial r}=v_{s}^{i} \square f_{s}(z) \sum_{m=-\infty}^{\infty} \lambda_{m s} \mathrm{e}^{\mathrm{i} m \theta} \text { on } \\
r_{i}=a_{i},-h \leq z \leq 0 \\
\frac{\partial \varphi_{R s-C}^{i}}{\partial z}=v_{s}^{i} \square g_{s}\left(r_{i}\right) \sum_{m=-\infty}^{\infty} \lambda_{m s} \mathrm{e}^{\mathrm{i} m \theta} \text { on } \\
z=-h, r_{i} \leq a_{i}
\end{gathered}
$$

where $v_{s}^{i}=-\mathrm{i} \omega_{0} \zeta_{s}^{i}$, is the velocity of cylinder $i$ in mode $s$. The expressions of $f_{s}(z), g_{s}\left(r_{i}\right), \lambda_{m s}$ are shown as follows:

$$
\begin{aligned}
& f_{s}(z)=1, \quad g_{s}\left(r_{i}\right)=0, s=1,2 \\
& f_{s}(z)=0, g_{s}\left(r_{i}\right)=1, s=3 \\
& f_{s}(z)=-(z-\bar{z}), g_{s}\left(r_{i}\right)=r_{i}, s=4 \\
& f_{s}(z)=(z-\bar{z}), \quad g_{s}\left(r_{i}\right)=-r_{i}, s=5 \\
& \lambda_{11}=\lambda_{15}=\frac{1}{2}, \lambda_{13}=0, \lambda_{12}=\lambda_{14}=\frac{1}{2 i} \\
& \lambda_{01}=\lambda_{05}=0, \lambda_{03}=1, \lambda_{02}=\lambda_{04}=0 \\
& \lambda_{-11}=\lambda_{-15}=\frac{1}{2}, \lambda_{-13}=0, \lambda_{-12}=\lambda_{-14}=-\frac{1}{2 i} \\
& \lambda_{m s}=0, m \neq 0, \pm 1
\end{aligned}
$$

The other boundary conditions on the free sur- 
face, the seabed and at infinity, the matching conditions on the interface between the exterior and core regions, and the Laplace's equation are listed as follows

$\nabla^{2} \varphi_{R s-E}^{i}=0$, in fluid domain

$\frac{\partial \varphi_{R s-E}^{i}}{\partial z}-\frac{\omega^{2}}{g} \varphi_{R s-E}^{i}=0, \quad z=0, \quad a_{i}<r_{i}<\infty$

$\frac{\partial \varphi_{R s-E}^{i}}{\partial z}=0, \quad z=-d, a_{i}<r_{i}<\infty$

$\lim _{r \rightarrow \infty} \sqrt{r}\left(\frac{\partial \varphi_{R s-E}^{i}}{\partial r}-\mathrm{i} k \varphi_{R s-E}^{i}\right)=0$

$\nabla^{2} \varphi_{R s-C}^{i}=0$, in fluid domain

$\frac{\partial \varphi_{R s-C}^{i}}{\partial z}=0, \quad z=-d, \quad 0 \leq r_{i} \leq a_{i}$

$\varphi_{R s-E}^{i}=\varphi_{R s-C}^{i}, \quad r_{i}=a_{i}, \quad-d \leq z \leq-h$

$\frac{\partial \varphi_{R s-E}^{i}}{\partial r}=\frac{\partial \varphi_{R s-C}^{i}}{\partial r}, \quad r_{i}=a_{i}, \quad-d \leq z \leq-h$

$\frac{\partial \varphi_{R s-E}^{i}}{\partial r}=v_{s}^{i} f_{s}(z) \sum_{m=-\infty}^{\infty} \lambda_{m s} \mathrm{e}^{\mathrm{i} m \theta}, r_{i}=a_{i}, \quad-h \leq z \leq 0$

The radiation potential in the exterior region of cylinder $i$, in the $s$ th mode oscillation is

$$
\begin{gathered}
\varphi_{R s-E}^{i}=-\mathrm{i} \omega_{0} \zeta_{s}^{i} \sum_{m=-\infty}^{\infty}\left[R_{0 m s}^{i} Z_{0}(z) H_{m}\left(k_{0} r_{i}\right)+\right. \\
\left.\sum_{n=1}^{\infty} R_{n m s}^{i} Z_{n}(z) K_{m}\left(k_{n} r_{i}\right)\right] \mathrm{e}^{\mathrm{i} m \theta_{i}}
\end{gathered}
$$

where $H_{m}, K_{m}$ are the $m$ th-order Hankel functions of the first kind and the modified Bessel functions of the second kind, respectively. The wave numbers $k_{0}$, $k_{n}(n=1,2, \cdots)$ are the positive real roots of the following dispersion equations

$$
\begin{aligned}
& k_{0} \tanh k_{0} d=\frac{\omega_{0}^{2}}{g} \\
& k_{n} \tan k_{n} d=-\frac{\omega_{0}^{2}}{g}, n=1,2, \cdots
\end{aligned}
$$

while $Z_{0}(z)$ and $Z_{n}(z), R_{0 m s}^{i}$ and $R_{n m s}^{i}$ are expressed as:

$Z_{0}(z)=\frac{\cosh k_{0}(z+d)}{\cosh k_{0} d}, n=0$

$Z_{n}(z)=\cos k_{n}(z+d), n \geq 1$

$R_{0 m s}^{i}=\frac{D_{0 m}^{s} \cosh k_{0} d}{H_{m}^{\prime}\left(k_{0} a_{i}\right) N_{0}^{1 / 2}}, m= \pm 1, n=0$

$R_{n m s}^{i}=\frac{D_{n m}^{s}}{K_{m}^{\prime}\left(k_{n} a_{i}\right) N_{n}^{1 / 2}}, m= \pm 1, n>0$

$D_{0 m}^{s}$ and $D_{n m}^{s}$ can be solved from Eq.(A1) in the appendix, and

$$
N_{0}=\frac{1}{2}\left(1+\frac{\sinh 2 k_{0} d}{2 k_{0} d}\right), N_{n}=\frac{1}{2}\left(1+\frac{\sin 2 k_{n} d}{2 k_{n} d}\right)
$$

Equation (9) can be expressed in matrix form as

$\varphi_{R s-E}^{i}=-\mathrm{i} \omega_{0} \zeta_{s}^{i} \boldsymbol{R}_{i s}^{\mathrm{T}} \boldsymbol{\psi}_{i}^{D-E}=-\mathrm{i} \omega_{0} \zeta_{s}^{i} \boldsymbol{R}_{i s}^{\mathrm{T}} \boldsymbol{T}_{i j} \boldsymbol{\psi}_{j}^{I}$

where $\boldsymbol{R}_{i s}(0, m)=R_{0 m s}^{i}, \quad \boldsymbol{R}_{i s}(n, m)=R_{n m s}^{i}$, and the column vector of the partial diffraction waves in the exterior region $\psi_{i}^{D-E}$ is

$\psi_{i}^{D-E}(n, m)=Z_{0}(z) H_{m}\left(k_{0} r_{i}\right) \mathrm{e}^{\mathrm{i} m \theta_{i}}, \quad n=0$

$\boldsymbol{\psi}_{i}^{D-E}(n, m)=Z_{n}(z) K_{m}\left(k_{n} r_{i}\right) \mathrm{e}^{\mathrm{i} m \theta_{i}}, \quad n \geq 1$

By using the Graf's addition theorem for Bessel functions, we have

$$
\begin{gathered}
\psi_{i}^{D-E}(n, m)=\sum_{l=-\infty}^{\infty} H_{m-l}\left(k_{0} R_{i j}\right) \mathrm{e}^{\mathrm{i} \alpha_{i j}(m-l)} \square \\
Z_{0}(z) J_{l}\left(k_{0} r_{j}\right) \mathrm{e}^{\mathrm{i} l \theta_{j}}, n=0 \\
\psi_{i}^{D-E}(n, m)=\sum_{l=-\infty}^{\infty} K_{m-l}\left(k_{n} R_{i j}\right) \mathrm{e}^{\mathrm{i} \alpha_{i j}(m-l)}(-1)^{l} \square \\
Z_{n}(z) I_{l}\left(k_{n} r_{j}\right) \mathrm{e}^{\mathrm{i} l \theta_{j}}, n \geq 1
\end{gathered}
$$

which can also be expressed in matrix form as 
$\boldsymbol{\psi}_{i}^{D-E}=\boldsymbol{T}_{i j} \boldsymbol{\psi}_{j}^{I}$

where $\boldsymbol{T}_{i j}$ defines a coordinate transformation from $i$ to $j$, which means that the waves emanate from cylinder $i$ in the local coordinate system centered in the origin of cylinder $j$. The elements in $\boldsymbol{T}_{i j}$ are

$$
\begin{aligned}
& \boldsymbol{T}_{i j}(n, m, l)=H_{m-l}\left(k_{0} R_{i j}\right) \mathrm{e}^{\mathrm{i} \alpha_{i j}(m-l)}, n=0 \\
& \boldsymbol{T}_{i j}(n, m, l)=K_{m-l}\left(k_{n} R_{i j}\right) \mathrm{e}^{\mathrm{i} \alpha_{i j}(m-l)}(-1)^{l}, n \geq 1
\end{aligned}
$$

$\psi_{j}^{I} \quad$ is the column vector of incident partial-wave functions

$$
\begin{aligned}
& \psi_{j}^{I}(n, m)=Z_{0}(z) J_{m}\left(k_{0} r_{j}\right) \mathrm{e}^{\mathrm{i} m \theta_{j}}, \quad n=0 \\
& \boldsymbol{\psi}_{j}^{I}(n, m)=Z_{n}(z) I_{m}\left(k_{n} r_{j}\right) \mathrm{e}^{\mathrm{i} m \theta_{j}}, \quad n \geq 1
\end{aligned}
$$

The exterior potential in the vicinity of cylinder $i$, which is generated from the diffraction of waves emanating from other cylinders by the cylinder $i$, is:

$$
\begin{array}{r}
\varphi_{D 1-E}^{i}=\sum_{m=-\infty}^{\infty}\left[A_{R 0 m}^{i} Z_{0}(z) H_{m}\left(k_{0} r_{i}\right)+\right. \\
\left.\sum_{n=1}^{\infty} A_{R n m}^{i} Z_{n}(z) K_{m}\left(k_{n} r_{i}\right)\right] \mathrm{e}^{\mathrm{i} m \theta_{i}}
\end{array}
$$

and in matrix form

$$
\varphi_{D 1-E}^{i}=\boldsymbol{A}_{R i}^{\mathrm{T}} \boldsymbol{\psi}_{i}^{D}=\boldsymbol{A}_{R i}^{\mathrm{T}} \boldsymbol{T}_{i j} \boldsymbol{\psi}_{j}^{I}
$$

where $\boldsymbol{A}_{R i}$ is the unknown column vector consisting of amplitudes of diffraction partial waves.

The total incident potential in the vicinity of cylinder $j$ is the sum of the radiation potentials due to the different oscillations of other $N-1$ columns and the diffraction potentials resulting from the hydrodynamic interactions between cylinders, i.e.,

$$
\begin{aligned}
\varphi_{j}^{I} & =\left.\sum_{i=1, i \neq j}^{N} \varphi_{R s-E}^{i}\right|_{j}+\left.\sum_{i=1, i \neq j}^{N} \varphi_{D 1-E}^{i}\right|_{j}= \\
& \sum_{i=1, i \neq j}^{N} \sum_{s=1}^{5}\left(-\mathrm{i} \omega_{0} \zeta_{s}^{i} \boldsymbol{R}_{i s}^{\mathrm{T}}\right) T_{i j} \psi_{j}^{I}+\sum_{i=1, i \neq j}^{N} \boldsymbol{A}_{R i}^{\mathrm{T}} \boldsymbol{T}_{i j} \boldsymbol{\psi}_{j}^{I}= \\
& \sum_{i=1, i \neq j}^{N}\left[\sum_{s=1}^{5}\left(-\mathrm{i} \omega_{0} \zeta_{s}^{i} \boldsymbol{R}_{i s}^{\mathrm{T}}\right)+\boldsymbol{A}_{R i}^{\mathrm{T}}\right] \boldsymbol{T}_{i j} \boldsymbol{\psi}_{j}^{I}
\end{aligned}
$$

For cylinder $j$, the diffraction potential $\varphi_{D 1-E}^{i}$ and the incident potential $\varphi_{j}^{I}$ can be related by the diffraction transfer matrix $B_{j}^{E}$ in the exterior region, as

$$
\boldsymbol{A}_{R j}=\boldsymbol{B}_{j}^{E} \sum_{i=1, i \neq j}^{N} \boldsymbol{T}_{i j}^{\mathrm{T}}\left[\sum_{s=1}^{5}\left(-\mathrm{i} \omega_{0} \zeta_{s}^{i} \boldsymbol{R}_{i s}\right)+\boldsymbol{A}_{R i}\right]
$$

where $\boldsymbol{B}_{j}^{E}$ is obtained from the results of the diffraction by a single truncated circular cylinder due to the unit-amplitude incidence of the progressive and the evanescent cylindrical waves. The elements of $\boldsymbol{B}_{j}^{E}$ are listed in the appendix.

With the known $\boldsymbol{B}_{j}^{E}$ and $\boldsymbol{R}_{i s}$, the unknown vector $\boldsymbol{A}_{R j}$ can then be obtained by solving Eq.(22), when $\zeta_{s}^{i}$ is prescribed. Thus the total exterior potential in the vicinity of cylinder $j$ due to the oscillations of each cylinder with different modes $(s=1,2,3,4,5)$ is obtained as

$$
\begin{array}{r}
\varphi_{R D-E}^{j}=\left[\sum_{s=1}^{5}\left(-\mathrm{i} \omega_{0} \zeta_{s}^{j} \boldsymbol{R}_{j s}^{\mathrm{T}}\right)+\boldsymbol{A}_{R j}^{\mathrm{T}}\right] \boldsymbol{\psi}_{j}^{D-E}+ \\
\sum_{i=1, i \neq j}^{N}\left[\sum_{s=1}^{5}\left(-\mathrm{i} \omega_{0} \zeta_{s}^{j} \boldsymbol{R}_{i s}^{\mathrm{T}}\right)+\boldsymbol{A}_{R i}^{\mathrm{T}}\right] \boldsymbol{T}_{i j} \boldsymbol{\psi}_{j}^{I}
\end{array}
$$

Following the same procedure, the velocity potential of cylinder $j$ in its core region can be deduced as

$$
\begin{aligned}
\varphi_{R D-C}^{j} & =\sum_{s=1}^{5}\left[-\mathrm{i} \omega_{0} \zeta_{s}^{j} \varphi_{R s}^{C}\left(r_{j}, \theta_{j}, z\right)\right]+ \\
& \left\{\sum_{i=1, i \neq j}^{N}\left[\sum_{s=1}^{5}\left(-\mathrm{i} \omega_{0} \zeta_{s}^{i} \boldsymbol{R}_{i s}^{\mathrm{T}}\right)+\boldsymbol{A}_{R i}^{\mathrm{T}}\right] \boldsymbol{T}_{i j}\right\}\left(\boldsymbol{B}_{j}^{C}\right)^{\mathrm{T}} \boldsymbol{\psi}_{j}^{D-C}
\end{aligned}
$$

where $\varphi_{R s}^{C}(r, \theta, z)$ is the potential of cylinder $j$ in its core region due to the unit-velocity oscillations of this cylinder as an isolated one (not as the component of the array of cylinders)

$$
\begin{gathered}
\varphi_{R s}^{c}\left(r_{j}, \theta_{j}, z\right)=\sum_{m=-\infty}^{\infty}\left\{\frac{C_{0 m}^{s}}{2}\left(\frac{r_{j}}{a}\right)^{|m|}+\Lambda_{s} \lambda_{m s}+\right. \\
\left.\sum_{n=1}^{\infty} C_{n m}^{s} \frac{I_{m}\left(\frac{n \pi r_{j}}{d-h}\right)}{I_{m}\left(\frac{n \pi a}{d-h}\right)} \cos \left[\frac{n \pi(z+d)}{d-h}\right]\right\} \mathrm{e}^{\mathrm{i} m \theta_{j}}
\end{gathered}
$$


$\Lambda_{s}=0, s=1,2$

$\Lambda_{s}=\frac{1}{2(d-h)}\left[(z+d)^{2}-\frac{r_{j}^{2}}{2}\right], s=3$

$\Lambda_{s}=\frac{r_{j}}{2(d-h)}\left[(z+d)^{2}-\frac{r_{j}^{2}}{4}\right], s=4$

$\Lambda_{s}=-\frac{1}{2(d-h)}\left[(z+d)^{2}-\frac{r_{j}^{2}}{4}\right], s=5$

$C_{0 m}^{s}$ and $C_{0 m}^{s}$ in Eq.(25) can be found in the appendix.

$\boldsymbol{\psi}_{j}^{D-C}$ in Eq.(24) is the column vector of the partial-wave functions in the core region

$\boldsymbol{\psi}_{j}^{D-C}(p, m)=r_{j}^{|m|} \mathrm{e}^{\mathrm{i} m \theta_{j}}, \quad p=0$

$\psi_{j}^{D-C}(p, m)=I_{m}\left(\frac{p \pi r_{j}}{d-h}\right) \mathrm{e}^{\mathrm{i} m \theta_{j}}, \quad p \geq 1$

$\boldsymbol{B}_{j}^{C}$ in Eq.(24) is the diffraction transfer matrix for cylinder $j$ in its core region, whose elements are listed in the appendix.

Therefore, the velocity potential in the vicinity of cylinder $j$ as each cylinder in the array oscillates in an arbitrary mode $s(s=1-5)$ or their combinations with an amplitude $\zeta_{s}^{i}(i=1,2, \ldots, N)$ is obtained. $\varphi_{R D-E}^{j}$ is the potential for the exterior region adjacent to cylinder $j$, which is given in Eq.(23). $\varphi_{R D-C}^{j}$ is the potential for the core region of cylinder $j$, which is given in Eq.(24).

\section{The hydrodynamic forces}

When $\varphi_{R D-E}^{j}$ and $\varphi_{R D-C}^{j}$ are obtained, the firstorder pressure on the surface of cylinder $j$ can then be obtained as

$$
p_{R D}^{j}=\operatorname{Re}\left\{-\left.\rho \frac{\partial \Phi_{R D}^{j}}{\partial t}\right|_{r_{j}=a_{j}}\right\}
$$

The pressure integration over the wetted surface of cylinder $j$ yields the resultant first-order hydrodynamic forces $\Gamma^{j}$ and moments $\chi^{j}$

$\Gamma^{j}=\int p^{j} \boldsymbol{n}^{j} \mathrm{~d} S$ $\boldsymbol{\chi}^{j}=\int p^{j}\left(\boldsymbol{L} \times \boldsymbol{n}^{j}\right) \mathrm{d} S$

The space and time factors of $\Gamma^{j}$ and $\chi^{j}$ can also be separated as

$$
\begin{aligned}
& \boldsymbol{\Gamma}^{j}=\operatorname{Re}\left\{\boldsymbol{F}^{j} \mathrm{e}^{-\mathrm{i} \omega_{0} t}\right\} \\
& \chi^{j}=\operatorname{Re}\left\{\boldsymbol{M}^{j} \mathrm{e}^{-\mathrm{i} \omega_{0} t}\right\}
\end{aligned}
$$

where $\boldsymbol{n}^{j}$ is the unit normal vector on cylinder $j$ pointing out of the fluid domain, $\boldsymbol{L}$ is the position vector of a point on the body surface with respect to the center of gravity of cylinder $j$.

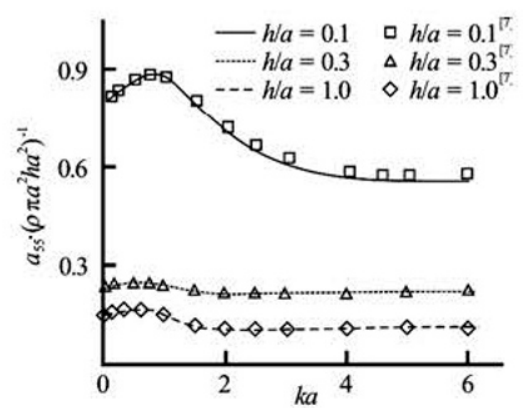

(a)

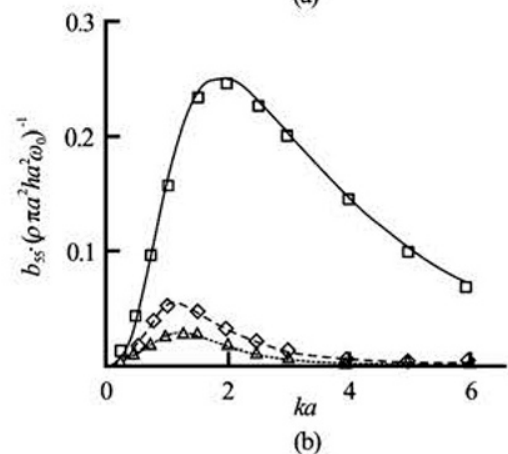

Fig.3 Added masses and damping coefficients for $d / a=3$

\section{Results for several cases and discussions}

Based on the method developed in the preceding sections, a computer program is developed. The program is verified before further calculations for the hydrodynamic interactions between each oscillating body in the array of cylinders.

The radiation hydrodynamic coefficients of a single oscillating truncated cylinder were given in Ref.[7]. Several examples in Ref.[7] are recalculated, and the results are shown in Fig.3 and Fig.4. $a_{s p}, b_{s p}$ are the added mass and the damping coefficients of a single cylinder, respectively, where $p$ denotes the oscillation mode and $s$ the direction of the force/ moment. 


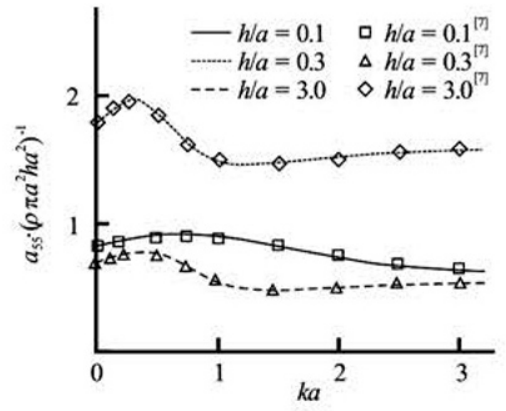

(a)

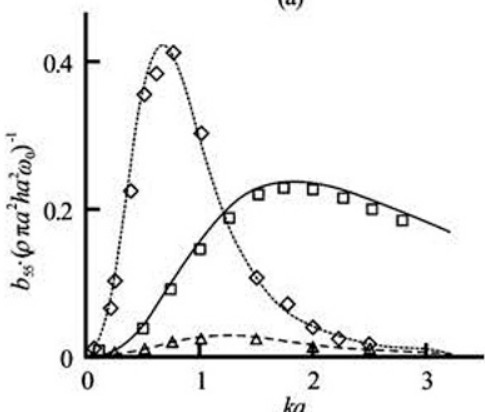

(b)

Fig.4 Added masses and damping coefficients for $d / a=10$

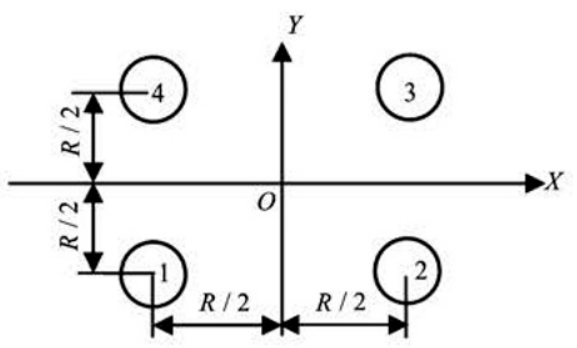

Fig. 5 Sketch of an array of four cylinders

In Figs.3-4, various lines such as solid lines, dash-dot lines etc. denote the results of this paper, the points with various shape denote the results of Ref.[7]. It can be seen that the agreement is good. Furthermore, an array of four truncated circular cylinders is analyzed, as shown in Fig.5.

Firstly, the array with $d=10 a, h=5 a, R=$ $50 a$ is calculated, where $a$ is the radius of a cylinder, and $R$ is the spacing. Because of the spacing between cylinders are very large, the case can be taken as the limit case with the spacing tending to infinity. Thus the interactions between the cylinders in the array are small, and the results should be close to those of a single isolated cylinder. Our results indicate that this is indeed the case. The added mass and damping coefficients of Cylinder 1 are calculated, as Cylinder 1 oscillates in surge, heave and pitch modes with other cylinders held in rest. As shown in Fig.6, the results of Cylinder 1 in the array agree with those of a single isolated cylinder. In Fig.6, the points with various

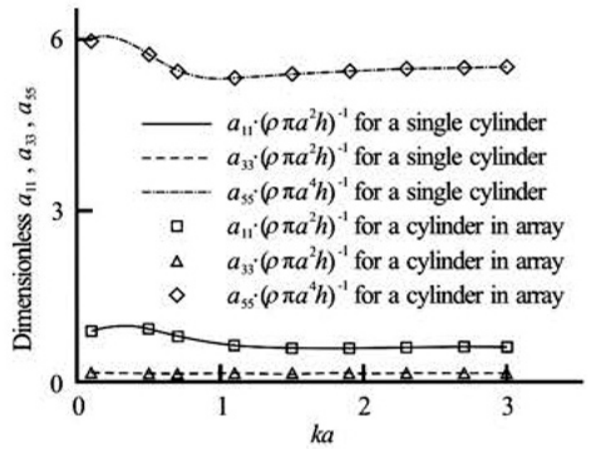

(a)

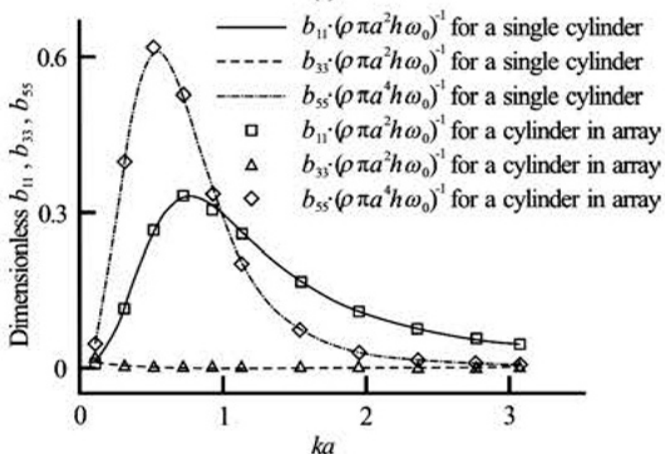

(b)

Fig.6 Comparisons of added mass and damping coefficients between an isolated cylinder and a cylinder in an array with large spacing

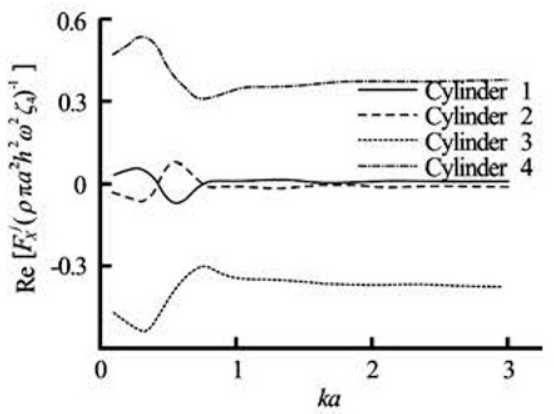

(a)

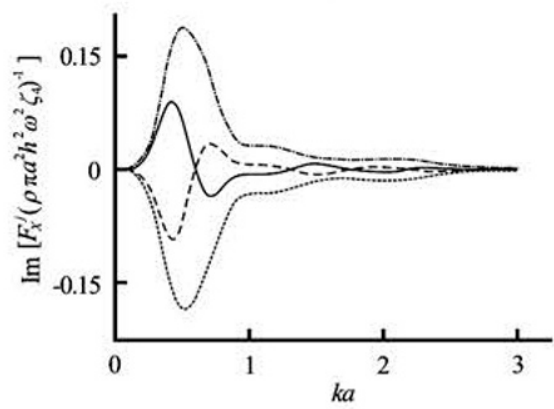

(b)

Fig.7 Cylinders 1, 2, 3, $4 X-(1$ and 2 roll in phase, 3 and 4 pitch out of phase)

shapes denote the results of a cylinder in the array, and the various lines denote those of a single cylinder. 


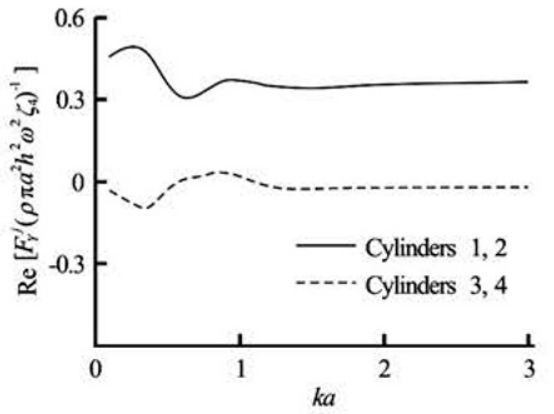

(a)

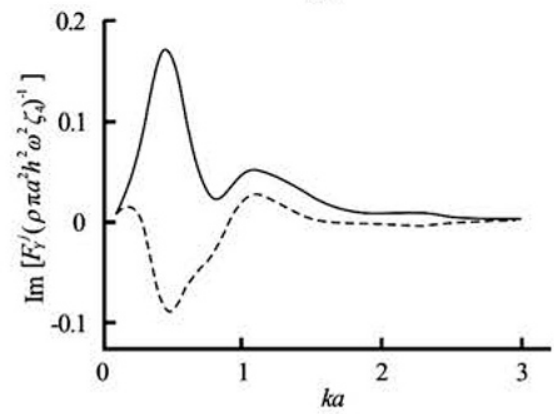

(b)

Fig. 8 Cylinders 1, 2, 3, $4 Y$ - (1 and 2 roll in phase, 3 and 4 pitch out of phase)

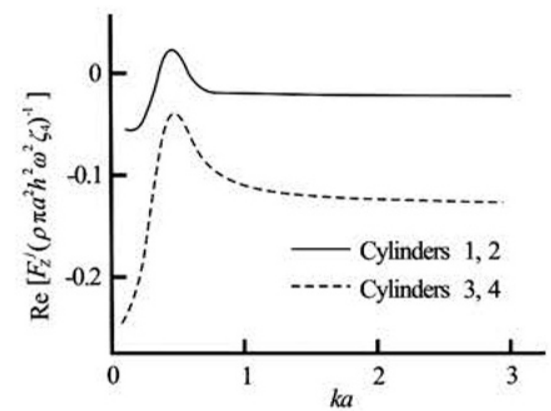

(a)

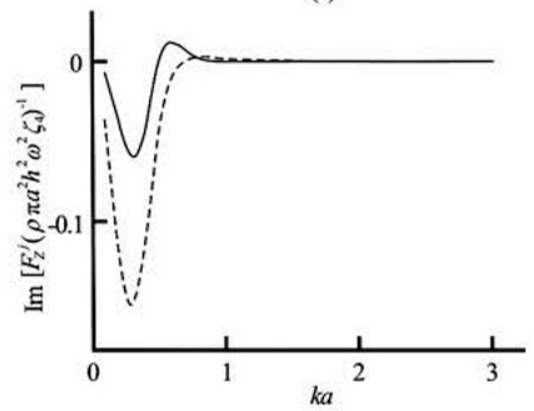

(b)

Fig.9 Cylinders 1, 2, 3, $4 Z$ - (1 and 2 roll in phase, 3 and 4 pitch out of phase)

Secondly, another array with $d=20 a, h=5 a$, $R=5 a$ is analyzed. For this cylinder array, two oscillation cases are considered. Case 1: Cylinders 1 and 2 oscillate in phase in the roll mode, at the same time, Cylinders 3 and 4 oscillate out of phase in the pitch mode. And the amplitudes of oscillations are the same. The hydrodynamic forces $\left(F_{x_{j}}^{j}, F_{y_{j}}^{j}, F_{z}^{j}\right)$ and moments $\left(M_{x_{j}}^{j}, M_{y_{j}}^{j}, 0\right)$ are calculated.

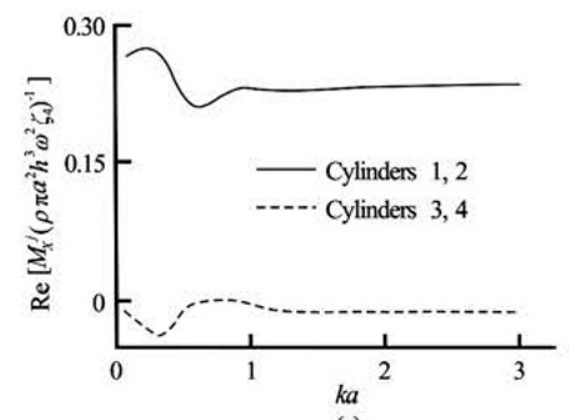

(a)

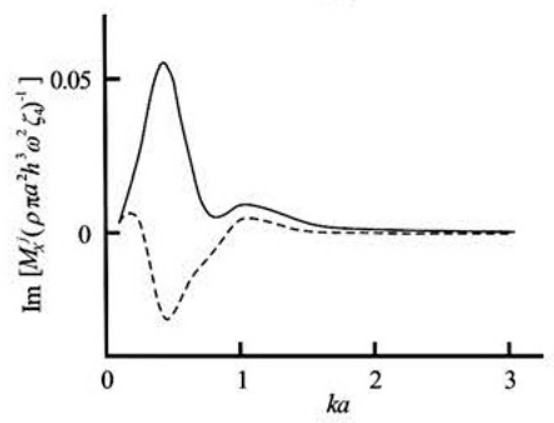

(b)

Fig. 10 Cylinders 1, 2, 3, 4 roll - (1 and 2 roll in phase, 3 and 4 pitch out of phase)

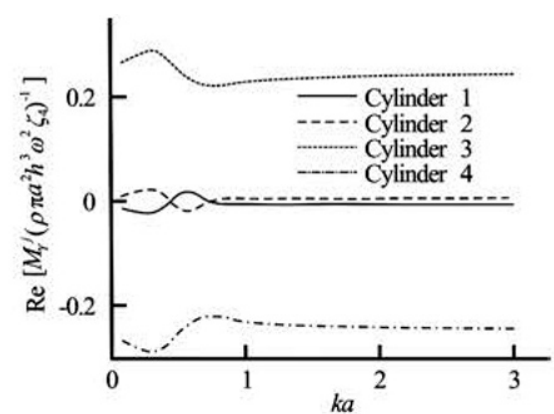

(a)

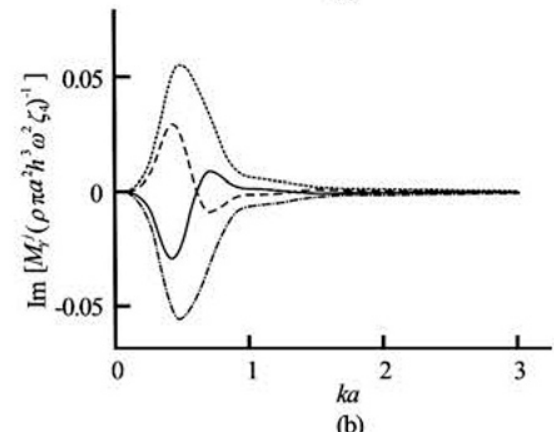

Fig. 11 Cylinders 1, 2, 3, 4 pitch - (1 and 2 roll in phase, 3 and 4 pitch out of phase)

Numerical results for Case 1 are shown in Figs.7 through 11. In the captions of these figures, " $X-$ ", 
" $Y$ - ", etc. denote the hydrodynamic forces or moments. For example, the label " $X$-" refers to the forces in $X$ direction on Cylinders 1, 2, 3, 4 due to combination oscillations of in phase roll of Cylinders 1 and 2 and out of phase pitch of Cylinders 3 and 4 .

Because the geometry of this array of four circular cylinders is symmetric, and the oscillation modes of cylinders are symmetric or anti-symmetric, it can be deduced that: (1) the surge forces acting on Cylinders 1 and 2, Cylinders 3 and 4, should be symmetric about $Y$ axis, (2) the sway and heave forces on Cylinder 1 and Cylinder 3 should be equal to those on Cylinder 2 and Cylinder 4, respectively, (3) the roll moments on Cylinder 1 and Cylinder 3 should be equal to those on Cylinder 2 and Cylinder 4, respectively, (4) the pitch moments on Cylinders 1 and 2, Cylinders 3 and 4, should be symmetric about $Y$ axis. From Figs. 7 through 11, it can be seen that our results are in agreement with these observations. Comparing Fig.7 with Fig.11, and Fig.8 with Fig.10, it is seen that the variation trends versus $k a$ of the surge forces and the pitch moments, the sway forces and the roll moments are similar.

From Figs. 7 through 11, it can also seen that the curves of the hydrodynamic forces and the moments fluctuate to different extents when $k a$ is smaller than 1.5 , and the curves tend to be flattened when $k a$ becomes larger than 1.5 .

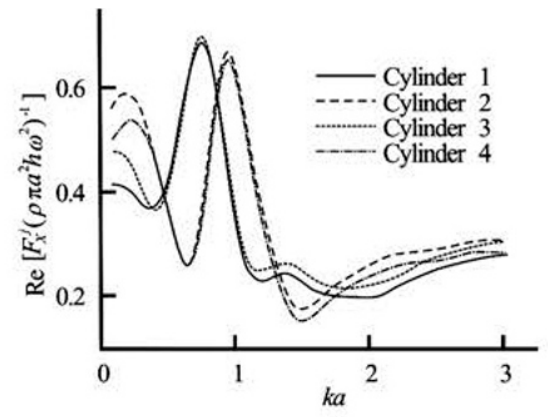

(a)

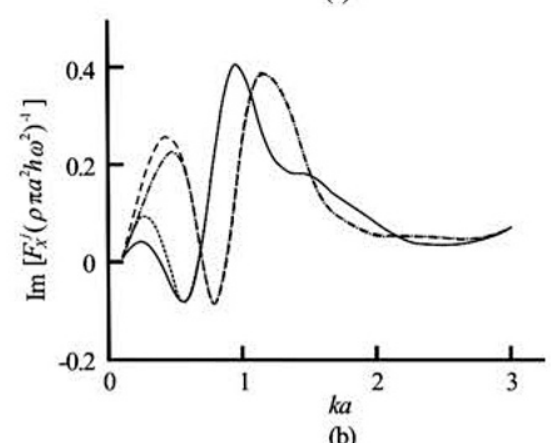

(b)

Fig.12 Cylinders 1, 2, 3, $4 \quad X$ - (cylinders 1-4 oscillate in five different modes simultaneously)

We now move on to Case 2. In Case 2, Cylinders 1-4 oscillate in five different modes simultaneously. The amplitudes of oscillations are given as: $\zeta_{s}^{j}=1$, $s=1,2,3, h \zeta_{s}^{j}=1, s=4,5$.

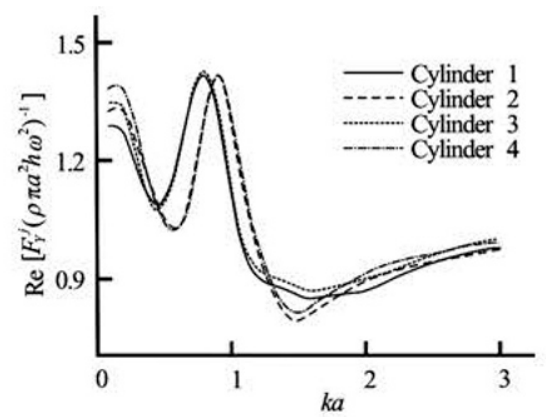

(a)

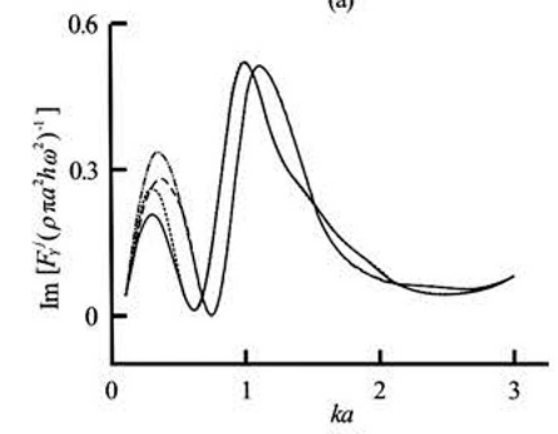

(b)

Fig.13 Cylinders 1, 2, 3, $4 Y$-(cylinders 1-4 oscillate in five different modes simultaneously)

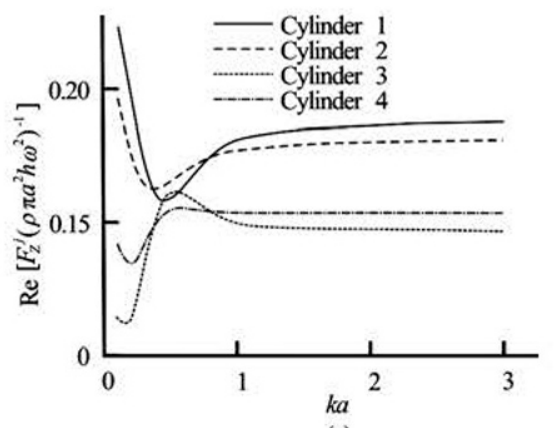

(a)

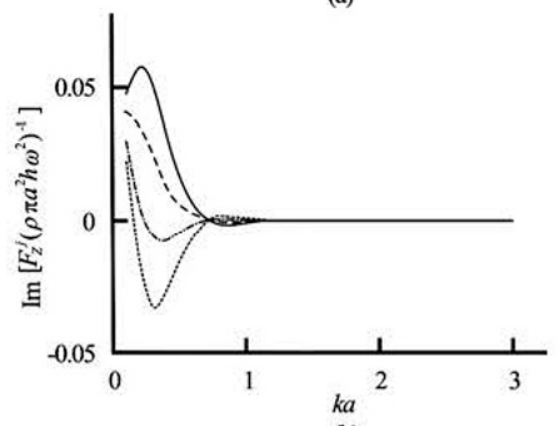

(b)

Fig.14 Cylinders 1, 2, 3, 4 Z - (cylinders 1-4 oscillate in five different modes simultaneously)

Numerical results for Case 2 are shown in Figs.12 through 16. In the captions of these figures, " $X-$-", " $Y$ - " etc. denote the hydrodynamic forces or moments. For example, the label " $X$-" refers to the 
forces in $X$ direction on Cylinders 1, 2, 3, 4 due to combination oscillations of Cylinders 1-4 in five different modes simultaneously. From Figs.12 through16, it can be seen that the curves of the hydrodynamic forces and the moments fluctuate to different extents when $k a$ is smaller than 1.5 , whereas the forces and moments tend to increase or decrease monotonically as $k a$ increases. This example also shows the favorable application prospect of the method developed in preceding sections.

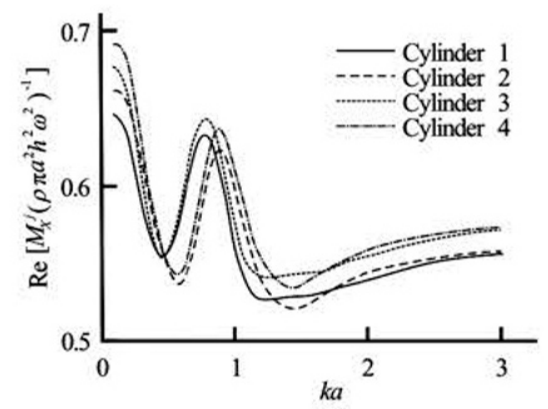

(a)

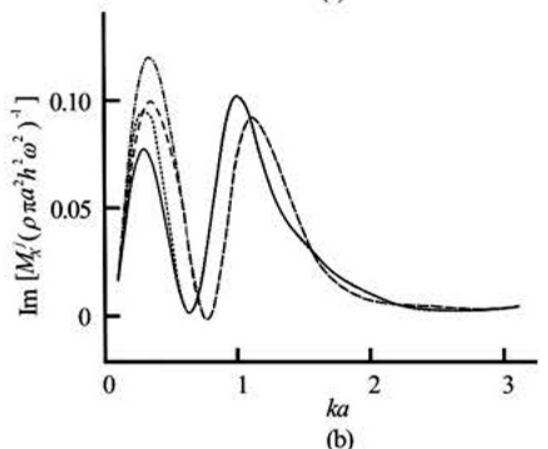

Fig.15 Cylinders 1, 2, 3, 4 roll - (cylinders 1-4 oscillate in five different modes simultaneously)

\section{Conclusions}

The hydrodynamic interactions of linear waves with arrays of circular truncated cylinders, in which each cylinder may be under any rigid oscillation modes (including translational and rotational modes with arbitrary prescribed amplitude), are investigated in this paper. An analytical method based on the eigenfunction expansion and the Graf's addition theorem for Bessel functions is developed. Formulas for velocity potentials, hydrodynamic forces and moments are derived, which are applicable to any number, spacing and dimension of cylinders.

Numerical results for typical arrays of truncated cylinders are obtained by using the proposed method. Results of degenerated cases show good agreement with the available results. Further results for arrays of cylinders, in which each cylinder oscillates with given amplitudes in all rigid modes such as surge, sway, heave, roll and pitch, are presented, which show the favorable application prospect of the method deve- loped in this paper.

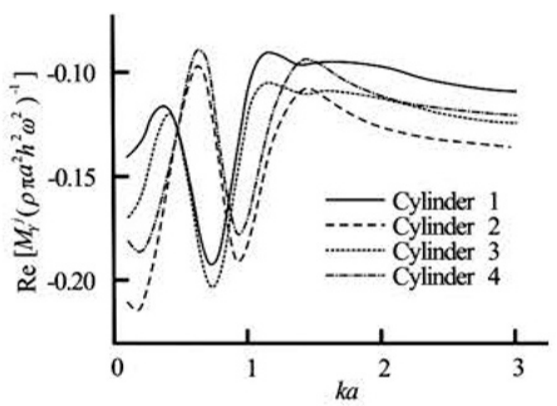

(a)

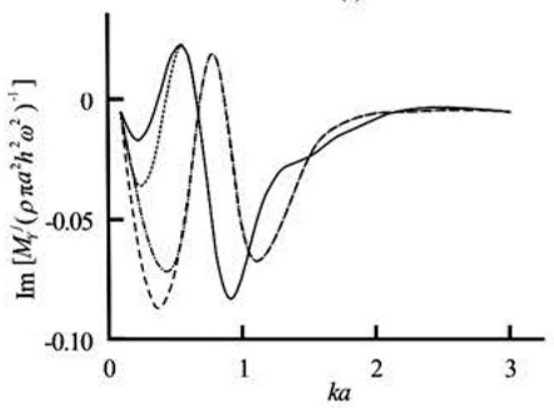

(b)

Fig.16 Cylinders 1, 2, 3, 4 pitch - (cylinders 1-4 oscillate in five different modes simultaneously)

\section{References}

[1] FALCAO A. F. O. Wave energy utilization: A review of the technologies[J]. Renewable and Sustainable Energy Reviews, 2010, 14(3): 899-918.

[2] ERIKSSON M., WATERS R. and SVENSSON O. et al. Wave power absorption: Experiments in open sea and simulation[J]. Journal of Applied Physics, 2007, 102(8): 084910.

[3] DONG Yan-qiu. Wave loads and response of the oilextraction platform in deep ocean[M]. Tianjin: Tianjin University Press, 2005(in Chinese).

[4] KASHIWAGI M. Hydrodynamic interactions among a great number of columns supporting a very large flexible structure[J]. Journal of Fluids and Structures, 2000, 14(7): 1013-1034.

[5] ZENG Xiao-hui, LI Xuan-wen and LIU Yang et al. Nonlinear dynamic responses of tension leg platform with slack-taut tether[J]. China Ocean Engineering, 2009, 23(1): 37-48.

[6] ZENG Xiao-hui, SHEN Xiao-peng and WU Ying-xiang Governing equations and numerical solutions of tension leg platform with finite amplitude motion[J]. Applied Mathematics and Mechanics (English Edition), 2007, 28(1): 37-49.

[7] SABUNCU T., CALISAL S. Hydrodynamic coefficients for vertical circular cylinder at finite depth[J]. Ocean Engineering, 1981, 8(1): 25-63.

[8] BHATTA D. D., RAHMAN M. On scattering and radiation problem for a circular cylinder in water of finite depth $[J]$. International Journal of Engineering Science, 2003, 41(9): 931-967.

[9] BHATTA D. D. Computations of hydrodynamic coefficients, displacement-amplitude ratios and forces for a 
floating cylinder due to wave diffraction and radiation[J]. International Journal of Non-Linear Mechanics, 2011, 46(8): 1027-1041.

[10] ZHENG Y. H., SHEN Y. M. and YOU Y. G. et al. Hydrodynamic properties of two vertical truncated cylinders in waves[J]. Ocean Engineering, 2005, 32(3-4): 241-271.

[11] KAGEMOTO H., YUE D. K. P. Interactions among multiple three-dimensional bodies in water waves: An exact algebraic method[J]. Journal of Fluid Mechanics, 1986, 166: 189-209.

[12] YILMAZ O., INCECIK A. and BARLTROP N. Wave enhancement due to blockage in semisubmersible and TLP structures[J]. Ocean Engineering, 2001, 28(5): 471-490.

[13] MIAO Guo-ping, LI Yi-le and LIU Ying-zhong. Theoretical basis of the recursion formulas for diffraction problems of circular piles of extremely large number[J]. Journal of Shanghai Jiaotong University, 2000, 34(1): 24-29(in Chinese).

[14] DUCLOS G., CLEMENT A. H. Wave propagation through arrays of unevenly spaced vertical piles[J]. Ocean Engineering, 2004, 31(13): 1655-1668.

[15] WALKER D. A. G., EATOCK TAYLOR R. Wave diffraction from linear arrays of cylinders[J]. Ocean Engineering, 2005, 32(17-18): 2053-2078.

[16] WALKER D. A. G., EATOCK TAYLOR R. and TAYLOR P. H. et al. Wave diffraction and near-trapping by a multi-column gravity-based structure[J]. Ocean Engineering, 2008, 35(2): 201-229.

[17] SIDDORN P., EATOCK TAYLOR R. Diffraction and independent radiation by an array of floating cylinders[J]. Ocean Engineering, 2008, 35(13): 12891303.

\section{Appendix}

The unknown coefficients $C_{n m}^{s}, D_{n m}^{s}$ of velocity potentials can be obtained by the following equations

$$
\begin{aligned}
& C_{n m}^{s}+\sum_{q=0}^{\infty} F_{n q}^{m} D_{q m}^{s}=R_{s}^{n m} \\
& D_{n m}^{s}-\sum_{n=0}^{\infty} G_{q n}^{m} C_{n m}^{s}=S_{s}^{q m}
\end{aligned}
$$

Here the known coefficients in Eq.(A1) are:

$$
\begin{aligned}
F_{n 0}^{m} & =-\frac{2}{d-h} \int_{-d}^{-h} \frac{H_{m}\left(k_{0} a\right)}{N_{0}^{1 / 2} H_{m}{ }^{\prime}\left(k_{0} a\right)} \\
& \cosh k_{0}(z+d) \cos \left[\frac{n \pi(z+d)}{d-h}\right] \mathrm{d} z= \\
& -\frac{2 H_{m}\left(k_{0} a\right) k_{0}(d-h)(-1)^{n} \sinh k_{0}(d-h)}{H_{m}{ }^{\prime}\left(k_{0} a\right) N_{0}^{1 / 2}\left[k_{0}^{2}(d-h)^{2}+(n \pi)^{2}\right]}
\end{aligned}
$$

$$
\begin{aligned}
F_{n q}^{m} & =-\frac{2}{d-h} \int_{-d}^{-h} \frac{K_{m}\left(k_{n} a\right)}{N_{q}^{1 / 2} K_{m}^{\prime}\left(k_{n} a\right)} \square \\
& \cos k_{q}(z+d) \cos \left[\frac{n \pi(z+d)}{d-h}\right] \mathrm{d} z= \\
& -\frac{2 K_{m}\left(k_{q} a\right) k_{q}(d-h)(-1)^{n} \sin k_{q}(d-h)}{K_{m}^{\prime}\left(k_{q} a\right) N_{q}^{1 / 2}\left[k_{q}^{2}(d-h)^{2}-(n \pi)^{2}\right]}
\end{aligned}
$$

$$
\begin{aligned}
G_{00}^{(m)} & =\frac{1}{k_{0} d} \int_{-d}^{-h} \frac{|m|}{2 a} Z_{0}(z) \mathrm{d} z= \\
& \frac{m \square \sinh k_{0}(d-h)}{\sqrt{2} a d k_{0}^{2}\left[1+\frac{\sinh 2 k_{0} d}{2 k_{0} d}\right]^{1 / 2}}, \quad q=0, n=0
\end{aligned}
$$

$$
\begin{aligned}
G_{q 0}^{(m)} & =\frac{1}{k_{q} d} \int_{-d}^{-h} \frac{|m|}{2 a} Z_{q}(z) \mathrm{d} z= \\
& \frac{m \square \sin k_{q}(d-h)}{\sqrt{2} a d k_{q}^{2}\left[1+\frac{\sin 2 k_{q} d}{2 k_{q} d}\right]^{1 / 2}}, q \geq 1, n=0
\end{aligned}
$$

$$
\begin{array}{r}
G_{0 n}^{(m)}=\frac{1}{k_{0} d} \int_{-d}^{-h} \frac{n \pi}{d-h} \frac{I_{m}^{\prime}\left(\frac{n \pi a}{d-h}\right)}{I_{m}\left(\frac{n \pi a}{d-h}\right)} \\
\cos \left[\frac{n \pi(z+d)}{d-h}\right] Z_{0}(z) \mathrm{d} z=
\end{array}
$$

$$
\begin{gathered}
\frac{I_{m}^{\prime}\left(\frac{n \pi a}{d-h}\right) \sqrt{2} n \pi(d-h)(-1)^{n} \sinh k_{0}(d-h)}{I_{m}\left(\frac{n \pi a}{d-h}\right) d\left(1+\frac{\sinh 2 k_{0} d}{2 k_{0} d}\right)^{1 / 2}\left[k_{0}^{2}(d-h)^{2}+n^{2} \pi^{2}\right]}, \\
q=0, n \geq 1
\end{gathered}
$$

$$
G_{q n}^{(m)}=\frac{1}{k_{q} d} \int_{-d}^{-h} \frac{n \pi}{d-h} \frac{I_{m}^{\prime}\left(\frac{n \pi a}{d-h}\right)}{I_{m}\left(\frac{n \pi a}{d-h}\right)}
$$




$$
\begin{gathered}
\cos \left[\frac{n \pi(z+d)}{d-h}\right] Z_{q}(z) \mathrm{d} z= \\
\frac{I_{m}^{\prime}\left(\frac{n \pi a}{d-h}\right) \sqrt{2} n \pi(d-h)(-1)^{n} \sin k_{q}(d-h)}{I_{m}\left(\frac{n \pi a}{d-h}\right) d\left(1+\frac{\sin 2 k_{q} d}{2 k_{q} d}\right)^{1 / 2}\left[k_{q}^{2}(d-h)^{2}-n^{2} \pi^{2}\right]} \\
q \geq 1, n \geq 1 \\
R_{s}^{n m}=-\frac{2}{d-h} \int_{-d}^{-h} \lambda_{m s} \Lambda_{s}(a, z) \cos \left[\frac{n \pi(z+d)}{d-h}\right] \mathrm{d} z \\
S_{s}^{q m}=\frac{\lambda_{m s}}{k_{q} d} \int_{-d}^{-h} \frac{\partial \Lambda_{s}(a, z)}{\partial r} Z_{q}(z) \mathrm{d} z+ \\
\frac{\lambda_{m s}}{k_{q} d} \int_{-h}^{0} f_{s}(z) Z_{q}(z) \mathrm{d} z
\end{gathered}
$$

The diffraction transfer matrices of the truncated single cylinder $\boldsymbol{B}_{j}^{E}$ and $\boldsymbol{B}_{j}^{C}$ are obtained following the procedure given in Ref.[11], the element of $\boldsymbol{B}_{j}^{E}$ (or $\boldsymbol{B}_{j}^{C}$ ) is the amplitude of the $q$ th (or $p$ th) partial wave of the diffraction potential due to a single unitamplitude incidence of mode $n$ on cylinder $j$, which are listed below:

$$
\begin{aligned}
& B_{j}^{E}(0,0, m)=-\frac{J_{m}^{\prime}\left(k_{0} a\right)}{H_{m}^{\prime}\left(k_{0} a\right)}+\frac{D_{0}^{m} \cosh k_{0} d}{H_{m}^{\prime}\left(k_{0} a\right) N_{0}^{1 / 2} \mathrm{e}^{\mathrm{i} m(\pi / 2-\beta)}} \\
& n=0, q=0
\end{aligned}
$$

$$
B_{j}^{E}(q, 0, m)=\frac{D_{q}^{m}}{K_{m}^{\prime}\left(k_{q} a\right) N_{q}^{1 / 2} \mathrm{e}^{\mathrm{i} m(\pi / 2-\beta)}}, n=0, \quad q \geq 1
$$

$$
\begin{aligned}
& B_{j}^{E}(0, n, m)=\frac{D[n]_{0}^{m} \cosh k_{0} d}{H_{m}^{\prime}\left(k_{0} a\right) N_{0}^{1 / 2}, n \geq 1, q=0} \\
& B_{j}^{E}(q, n, m)=\frac{D[n]_{q}^{m}}{K_{l}^{\prime}\left(k_{q} a\right) N_{q}^{1 / 2}}, n \geq 1, q \geq 1, q \neq n \\
& B_{j}^{E}(q, n, m)=-\frac{I_{m}^{\prime}\left(k_{q} a\right)}{K_{m}^{\prime}\left(k_{q} a\right)}+\frac{D[n]_{q}^{m}}{K_{m}^{\prime}\left(k_{q} a\right) N_{q}^{1 / 2}} \\
& n \geq 1, q \geq 1, \quad q=n \\
& B_{j}^{C}(0,0, m)=\frac{C_{0}^{m}}{2 a^{|m|} \mathrm{i}^{m}}, n=0, p=0 \\
& B_{j}^{C}(p, 0, m)=\frac{C_{p}^{m}}{I_{m}\left(\frac{p \pi a}{d-h}\right) \mathrm{i}^{m}} \cos \frac{p \pi(z+d)}{d-h} \\
& n=0, p \geq 1
\end{aligned}
$$

$B_{j}^{C}(0, n, m)=\frac{C[n]_{0}^{m}}{2 a^{|m|}}, n \geq 1, \quad p=0$

$$
B_{j}^{C}(p, n, m)=\frac{C[n]_{p}^{m}}{I_{m}\left(\frac{p \pi a}{d-h}\right)} \cos \frac{p \pi(z+d)}{d-h},
$$

$$
n \geq 1, \quad p \geq 1
$$

The coefficients such as $C[n]_{p}^{m}, \quad D[n]_{q}^{m}$ are determined by linear algebraic equations having the same form as Eq.(A1), which can be found in Ref.[12] and not listed here in detail. 